\title{
openheart Risk stratification for major adverse cardiac events and ventricular tachyarrhythmias by cardiac MRI in patients with cardiac sarcoidosis
}

\author{
Masakazu Yasuda, ${ }^{1}$ Yoshitaka Iwanaga, ${ }^{1}$ Takao Kato, ${ }^{2}$ Toshiaki Izumi, ${ }^{2}$ \\ Yasutaka Inuzuka, ${ }^{3}$ Takashi Nakamura, ${ }^{1}$ Yuki Miyaji, ${ }^{1}$ Takayuki Kawamura, ${ }^{1}$ \\ Shigeru Ikeguchi, ${ }^{3}$ Moriaki Inoko, ${ }^{2}$ Takashi Kurita, ${ }^{1}$ Shunichi Miyazaki ${ }^{1}$
}

To cite: Yasuda $\mathrm{M}$ Iwanaga Y, Kato T, et al. Risk stratification for major adverse cardiac events and ventricular tachyarrhythmias by cardiac MRI in patients with cardiac sarcoidosis. Open Heart 2016;3:e000437 doi:10.1136/openhrt-2016000437

- Additional material is available. To view please visit the journal (http://dx.doi.org/ 10.1136/openhrt-2016000437).

MY and YI both authors contributed equally to this work.

Received 14 March 2016 Revised 28 May 2016 Accepted 30 June 2016

\section{CrossMark}

${ }^{1}$ Division of Cardiology, Kindai University Faculty of Medicine, Osakasayama, Japan

${ }^{2}$ Kitano Hospital, Osaka, Japan

${ }^{3}$ Shiga Medical Center for Adults, Moriyama, Japan

Correspondence to Dr Yoshitaka Iwanaga; yiwanaga@med.kindai.ac.jp

\section{ABSTRACT}

Background: The presence of myocardial fibrosis by cardiac MRI has prognostic value in cardiac sarcoidosis, and localisation may be equally relevant to clinical outcomes.

Objective: We aimed to analyse cardiac damage and function in detail and explore the relationship with clinical outcomes in patients with cardiac sarcoidosis using cardiac MRI.

Methods: We included 81 consecutive patients with cardiac sarcoidosis undergoing cardiac MR. Left ventricular mass and fibrosis mass were calculated, and localisation was analysed using a 17-segment model. Participants underwent follow-up through 2015, and the development of major adverse cardiac events including ventricular tachyarrhythmias was recorded.

Results: Increased left ventricular fibrosis mass was associated with increased prevalence of ventricular tachyarrhythmias $(p<0.001)$. When localisation was defined as the sum of late gadolinium enhancement in the left ventricular basal anterior and basal anteroseptal areas, or the right ventricular area, it was associated with ventricular tachyarrhythmias $(p<0.001)$. Kaplan-Meier analysis during a median follow-up of 22.1 months showed that both the mass and localisation groupings for fibrosis were significantly associated with major adverse cardiac events or ventricular tachyarrhythmias and that when combined, the risk stratification was better than for each variable alone $(p<0.001$, respectively). By Coxproportional hazard risk analysis, the localisation grouping was an independent predictor for the both. Conclusions: In patients with cardiac sarcoidosis, both fibrosis mass and its localisation to the basal anterior/ anteroseptal left ventricle, or right ventricle was associated with the development of major adverse cardiac events or ventricular tachyarrhythmias. Cardiac MR with late gadolinium enhancement may be useful for improving risk stratification in patients with cardiac sarcoidosis.

\section{INTRODUCTION}

Cardiac involvement in sarcoidosis occurs in $20-30 \%$ of patients in autopsy studies, ${ }^{12}$ and

\section{KEY QUESTIONS}

What is already known about this subject?

- The presence of myocardial fibrosis by cardiac MRI has prognostic value in cardiac sarcoidosis.

What does this study add?

- The fibrosis localisation to the basal anterior/anteroseptal left ventricle, or right ventricle is associated with the development of major adverse cardiac events or ventricular tachyarrhythmias.

How might this impact on clinical practice?

- Cardiac MRI with late gadolinium enhancement is promising for predicting adverse events including ventricular tachyarrhythmias.

has been closely associated with prognosis. ${ }^{3}$ A previous study reported that sudden cardiac death (SCD) occurred in two-thirds of patients with histological evidence of cardiac sarcoidosis (CS) ${ }^{5}$ Ventricular tachyarrhythmia (VA), resulting from myocardial granulomas and fibrosis that cause electric instability, has been identified as the underlying mechanism of SCD. The 5-year mortality rate in patients with CS when structural or functional abnormality is present, according to the diagnostic criteria of the Japanese Ministry of Health and Welfare (JMHW), has been reported at $40 \%$, with the cause of death being the progression of heart failure (HF) or VA. ${ }^{6}$ However, the factors related to the clinical manifestations and outcomes of CS remain unclear and the risk stratification and clinical management are difficult, particularly for VA and SCD.

Late gadolinium-enhancement cardiac MR (LGE-CMR) has been used to detect myocardial damage/fibrosis, and recent findings have suggested that it is more sensitive than clinical diagnostic criteria for detecting 
CS. $^{7} 8$ A recent study demonstrated that LGE-positive patients had a higher rate of adverse events (a Cox HR of 31.6 for death, aborted SCD, or appropriate ICD discharge), compared with LGE-negative patients in a cohort of patients with suspected CS. ${ }^{9}$ Although myocardial fibrosis indicated by LGE may predict potentially lethal events, the utility of LGE in patients with sarcoidosis who present with high-grade cardiovascular symptoms, such as HF, VA and advanced atrioventricular block (AVB), has not yet been established. In addition, detailed risk stratification in patients with CS using LGE-CMR remains challenging. Therefore, we analysed cardiac damage and function in detail and explored the relationship with clinical outcomes including VA, using CMR in patients with CS. We assumed that not only fibrosis mass but also its localisation might help to identify the risk of clinical outcomes in patients with CS.

\section{METHODS}

\section{Study protocol}

We enrolled consecutive patients with CS referred for CMR between 2008 and 2015 at three referral centers (Kindai University Hospital, Osakasayama, Japan; Kitano Hospital, Osaka, Japan; and Shiga Medical Center for Adults, Moriyama, Japan). The study was approved by the Institutional Review Boards.

Patients were enrolled if they fulfilled the following criteria: (1) definite CS: CS diagnosed by the 2006 revised JMHW criteria; ${ }^{10}$ or (2) suspected CS: strongly suspected CS based on a constellation of clinical characteristic manifestations and positive findings on echocardiography, 18F-fluorodeoxyglucose-positron emission tomography (FDG-PET), or CMR, with or without extra-CS, and after the exclusion of other known cardiac diseases. ${ }^{11}$ The characteristic positive findings of CMR and FDG-PET were based on those detailed in previous reports. ${ }^{7} 12$

\section{CMR image acquisition}

CMR was performed using a $1.5 \mathrm{~T}$ scanner (Intera 1.5T; Philips Medical Systems, the Netherlands), according to a standardised protocol. Cine images were acquired with a steady-state free-precession breath-hold sequence in three long-axis planes and contiguous short-axis slices $(10 \mathrm{~mm}$, no gap) from the atrioventricular ring to the apex. The visualisation of myocardial oedema was performed using a T2-weighted triple inversion recovery breath-hold pulse sequence. LGE images were obtained 10-15 min after intravenous administration of $0.2 \mathrm{mmol} /$ $\mathrm{kg}$ gadolinium-DTPA (Magnevist; Bayer Schering Pharma, Germany) using an inversion recovery gradient echo sequence with $5 \mathrm{~mm}$ slice thickness at the same position as the long-axis and short-axis cines at end-diastole. The inversion time was adjusted per patient to an optimal null signal from a normal myocardium, typically between 250 and $350 \mathrm{~ms}$.

\section{Image analysis}

$\mathrm{LV}$ volume, LV mass (LVM), and wall thickness were calculated with commercially available workstations (Aze Virtual Place; Aze , Japan) as previously described. ${ }^{13}$ The endocardial and epicardial borders of the LV myocardium were manually planimetered on successive short-axis cine images at end-diastole and LVM was derived by the summation-of-the-disks method and multiplication of the myocardial muscle volume by $1.05 \mathrm{~g} / \mathrm{cm}^{3}$. To assess LGE, all short-axis slices from the base to the apex were visually inspected to identify areas of normal (completely nulled) myocardium. The mean signal intensity (and SD) was derived, and a threshold of $6 \mathrm{SD}$ above the mean was used to define the areas of LGE. ${ }^{14}$ Summing the planimetered areas of LGE in all short-axis slices yielded the total volume (LV fibrosis mass; g), which was also expressed as a proportion of total LV myocardium (\% LGE).

Local wall thickness, LGE, and T2-weighted images were also analysed based on the American Heart Association's (AHA) 17-segment model. To evaluate the relationship between localisation of LGE and clinical outcomes, a localisation score (LS) was defined as the summation of LGE enhancement in segments identified by LGE localisation analysis. All analyses were performed by an experienced specialist, and reviewed and confirmed by a second expert. Both independent observers were blinded to the patients' identities and clinical profiles. Any discrepancies in analysis were adjudicated by a senior observer.

\section{Clinical follow-up}

Long-term clinical follow-up was for 50 months after the CMR testing and accomplished by patient-completed questionnaire, telephone interview or chart review. Major advanced cardiac events (MACE) were defined as the combination of cardiac mortality, admission for worsening HF, advanced AVB and VA. VA was defined as $>3$ consecutive ventricular contractions, appropriate implantable cardioverter defibrillator (ICD) discharge or ventricular fibrillation (VF). Sustained ventricular tachycardia (VT) was defined as a ventricular rhythm faster than $100 \mathrm{bpm}$ lasting at least $30 \mathrm{~s}$. All events were based on clinical diagnosis but were validated by reviewing the patients' medical records. Advanced AVB, VA or sustained VT was also confirmed by ECG or ICD records.

\section{Statistical analysis}

As part of the univariable analysis for continuous variables, comparisons among groups were performed with student's t-tests, one way analysis of variance, and Mann-Whitney tests. Pearson $\chi 2$ and the Fisher's exact test were used to assess differences in categorical variables. Cut-off levels of LV fibrosis mass index for VA events and the sensitivities and specificities of the cut-off levels were calculated using a receiver operating characteristics (ROC) curve analysis. Event-free survival curves were analysed using the Kaplan-Meier method 
and comparison between curves was carried out using log-rank tests. The multivariate analysis of clinical outcomes was evaluated with Cox's proportional hazard model, using JMP V.10.0 (SAS Institute, USA). HRs and $95 \%$ CIs were calculated. A $p$ value $<0.05$ was considered significant. All results are expressed as mean \pm SD.

\section{RESULTS}

\section{Baseline patient characteristics}

In total, 81 consecutive patients were enrolled, of which 35 were diagnosed as definite CS using the 2006 revised JMHW criteria and 46 were diagnosed as suspected CS clinically (table 1). Furthermore, 38 (46.9\%) patients had extracardiac involvements and $43(53.1 \%)$ patients were diagnosed as having isolated CS, with $31(38.3 \%)$ patients having prior VA events, $20(24.7 \%)$ having documented advanced AV block, and 24 (29.6\%) being complicated by HF. Plasma B-type natriuretic peptide $(<18.4 \mathrm{pg} / \mathrm{mL})$ and ACE inhibitors levels $(<21 \mathrm{IU} / \mathrm{L})$ were increased in $84.0 \%$ and $32.9 \%$ of the patients, respectively. Although just $15(18.5 \%)$ patients had taken corticosteroids at baseline, a further $27(33.3 \%)$ had taken them during follow-up.

\section{CMR findings and VA events}

In the CMR examination at baseline, a reduced $\mathrm{EF}$ $(<50 \%)$ was observed in $58.8 \%$ and LGE was present in $94.9 \%$, with lesions frequently observed in the basal septum (Segment 2: 75.9\%; Segment 3: 65.8\%) and RV $(57.0 \%)$. The mean \% LGE and FM index were $16.6 \%$ $\pm 12.8 \%$ and $11.3 \pm 9.6 \mathrm{~g} / \mathrm{m}^{2}$, respectively. During a median follow-up of 22.1 months, $25(30.7 \%)$ patients had VA episodes of which $8(9.9 \%)$ where new onset and the other $17(21.0 \%)$ where recurrences. Any VA event was observed in $39(48.1 \%)$ patients.

As shown in table 2, all VA events (ie, a history of VA and new-onset VA events) were highly associated with the existence of LGE in the basal anterior (segment 1) and basal anteroseptal (segment 2) LV, as well as the RV $(\mathrm{p}<0.01)$. Among patients with VA, almost all had LGE in segment 2 and LGE was more frequently present in segment 1 and the RV than in patients without VA. Accordingly, we defined the LS as the summation of the LGE in segment 1 , segment 2 , and the $\mathrm{RV}$, with a potential score of 0 to 3 . Figure 1 shows representative LGE images according to the AHA's 17-segment model and representative cases stratified by the LS. As shown in figure 2, when patients with all VA events were stratified by LS, LS was significantly associated with all VA events $(p<0.001)$. Increased LV FM index was also associated with all VA events $(p<0.001)$, and ROC analysis showed that the area under the ROC curve (AUC) for FM as an indicator of all VA events was $0.760(95 \%$ CI 0.651 to 0.870 ) (figure 2). The optimal cut-off value of LV FM index was $5.12 \mathrm{~g} / \mathrm{m}^{2}$, with a sensitivity of $86 \%$ and a specificity of $62 \%$.

Table 1 Baseline clinical characteristics and CMR findings

\begin{tabular}{|c|c|c|c|}
\hline & $\mathrm{N}=81$ & & \\
\hline Age, years & $62.9 \pm 13.0$ & Device implantation & \\
\hline Female & $49(60.5)$ & Pacemaker & $11(13.6)$ \\
\hline ACE, IU/L & $18.1 \pm 10.0$ & ICD/CRT-D & $8(9.9)$ \\
\hline $\mathrm{BNP}, \mathrm{pg} / \mathrm{mL}$ & $151.3 \pm 172.3$ & CMR parameters & \\
\hline Involvement & & LV EDV, mL & $157.7 \pm 61.7$ \\
\hline Lung & $20(24.7)$ & LV ESV, mL & $91.2 \pm 58.7$ \\
\hline Skin & $12(14.8)$ & LV EF, \% & $45.9 \pm 14.6$ \\
\hline Eye & $17(21.0)$ & LV mass index, $\mathrm{g} / \mathrm{m}^{2}$ & $68.2 \pm 19.1$ \\
\hline Other & $6(7.4)$ & T2 positive & $47(58.8)$ \\
\hline JMHW criteria, positive & $35(43.2)$ & $\%$ LGE area, \% & $16.6 \pm 12.9$ \\
\hline Medication & & Fibrosis mass index, $\mathrm{g} / \mathrm{m}^{2}$ & $11.3 \pm 9.6$ \\
\hline ACEi or ARB & $29(35.8)$ & LV morphological abnormality & \\
\hline$\beta$-blocker & 25 (30.9) & Thinning & $21(25.9)$ \\
\hline Diuretic & $26(32.1)$ & Aneurysmal & $13(16.0)$ \\
\hline Corticosteroid & $15(18.5)$ & & \\
\hline Antiarrhythmic drug & $15(18.5)$ & & \\
\hline \multicolumn{4}{|l|}{ Cardiac symptoms } \\
\hline NYHA functional class $\geq 1 \mathrm{I}$ & $24(29.6)$ & & \\
\hline Admission due to $\mathrm{HF}$ & $16(19.8)$ & & \\
\hline Syncope & $22(29.7)$ & & \\
\hline Atrial fibrillation & 10 (12.3) & & \\
\hline Advanced AV block & $20(24.7)$ & & \\
\hline VA & $31(42.5)$ & & \\
\hline \multicolumn{4}{|c|}{$\begin{array}{l}\text { Values are the mean } \pm \text { SD or number (\%). } \\
\text { ACEi, ACE inhibitor; ARB, angiotensin receptor blocker; AV, atrioventricular; BNP, B-type natriuretic peptide; CRT-D, cardiac } \\
\text { resynchronisation therapy-defibrillator; EDV, end-diastolic volume; EF, ejection fraction; ESV, end-systolic volume; HF, heart failure; HT, } \\
\text { hypertension; ICD, implantable cardioverter defibrillator; JMHW, Japanese Ministry of Health and Welfare; LGE, late gadolinium enhancement; } \\
\text { LV, left ventricular; NYHA. New York Heart Association; VA, ventricular tachyarrhythmia. }\end{array}$} \\
\hline
\end{tabular}


Table 2 Association of VA events with localisation of LGE according to the 17-segment AHA model

\begin{tabular}{llll}
\hline & LGE (\%) & & Fisher's test \\
\cline { 2 - 3 } AHA 17 segment & VA event (+) & VA event (-) & 0.0002 \\
\hline 1. Basal anterior & 63.2 & 22.0 & 0.0014 \\
2. Basal anteroseptal & 92.1 & 61.0 & 0.0015 \\
RV & 76.3 & 41.5 & 0.0238 \\
9. Mid inferoseptal & 55.3 & 29.3 & 0.0306 \\
12. Mid anterolateral & 34.2 & 12.2 & 0.0317 \\
3. Basal inferoseptal & 79.0 & 53.7 & 0.0372 \\
5. Basal inferolateral & 36.8 & 14.6 & 0.0542 \\
4. Basal inferior & 44.7 & 22.0 & 0.1070 \\
14. Apical septal & 31.6 & 14.6 & 0.1071 \\
15. Apical inferior & 21.1 & 7.3 & 0.1311 \\
7. Mid anterior & 36.8 & 19.5 & 0.1499 \\
10. Mid inferior & 42.1 & 24.4 & 0.1697 \\
8. Mid anteroseptal1 & 44.7 & 23.9 & 0.4204 \\
17. Apex & 10.5 & 4.9 & 0.4310 \\
6. Basal anterolateral & 29.0 & 19.5 & 0.5374 \\
13. Apical anterior & 18.4 & 12.2 & 1.0000 \\
11. Mid inferolateral & 21.1 & 19.5 & 1.0000 \\
16. Apical lateral & 13.2 & 14.6 & \\
\hline AHA, American Heart Association; LGE, late gadolinium enhancement; LV, left ventricle; RV, right ventricle; VA, ventricular tachyarrhythmias.
\end{tabular}

A LGE images by AHA 17 segment model
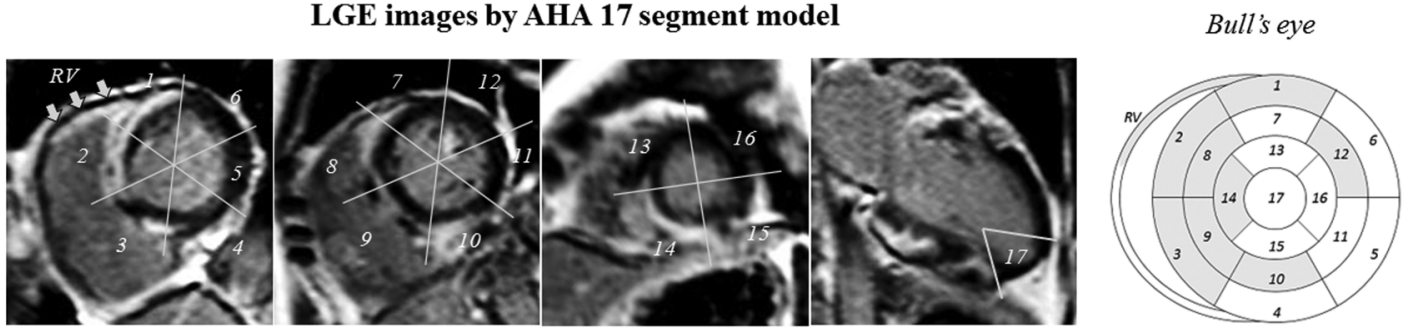

B

LGE images (localisation and mass)

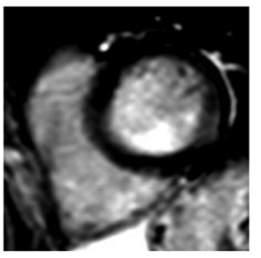

LS; 0

FM; 6.7

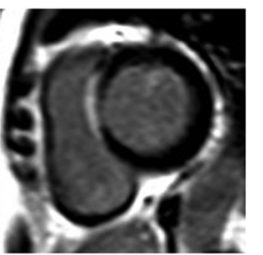

LS; $1(\operatorname{Seg} 2)$

FM; 14.7

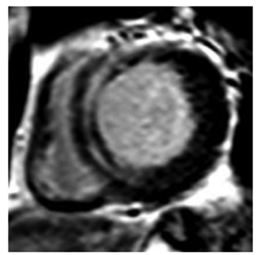

LS; 2 (Seg1, Seg2) FM; 45.6

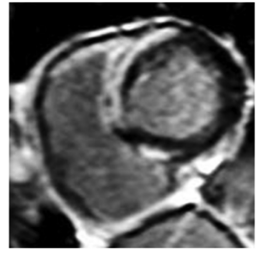

LS; 3 (Seg1, Seg2, RV) FM; 26.5
LS : Localisation score

$\mathrm{FM}:$ Fibrosis mass $(\mathrm{g})$

Figure 1 Representative LGE images using the AHA 17-segment model (A) and representative cases stratified by the LS (B). AHA, American Heart Association; FM, fibrosis mass; LGE, late gadolinium enhancement; LS, localisation score.

\section{Clinical outcome and risk stratification}

During a median follow-up of 22.1 months (lower quartile, 7.4 months; upper quartile, 38.1 months), 30 MACEs occurred: 3 patients died due to SCD or HF, 7 were admitted for worsening $\mathrm{HF}$ and 4 patients had advanced AVB. Twelve severe ventricular arrhythmias episodes such as sustained VT and VF occurred. In 10 patients, the origin was estimated by 12-lead ECG and 8 patients showed the ECG with inferior axis. They were from left/right ventricular outflow tract or high LV septum, which were associated with LS (the existence of LGE in segment 1, 2 or RV).
For the Kaplan-Meier analysis, patients were divided into two groups according to the optimal cut-off for $\mathrm{LV}$ FM index $\left(<5.12 \mathrm{~g} / \mathrm{m}^{2}(\mathrm{n}=29)\right.$ or $\left.\geq 5.12 \mathrm{~g} / \mathrm{m}^{2}(\mathrm{n}=45)\right)$, or the LS $(\leq 1 \quad(n=32)$ or $\geq 2(n=47))$. Patients with an LV FM $\geq 5.12 \mathrm{~g} / \mathrm{m}^{2}$ or an LS $\geq 2$ showed significantly worse prognoses in terms of both MACE and VA events (figure 3). In sustained VT or VF events, only increased LS was associated with worse prognosis $(\mathrm{p}=0.006)$. Lower EF $(<45 \%)$ was mildly associated with the worse prognosis in the MACE $(\mathrm{p}=0.045)$, and extracardiac sarcoidosis involvement was not associated with the both prognoses ( $\mathrm{p}=0.330$ and $\mathrm{P}=0.930$, respectively). 
Figure 2 Relationship between LV fibrosis mass index or localisation score and VA events. Stratification of patients with VA events by localisation score (A). Receiver operating characteristic (ROC) curves for LV fibrosis mass index as an indicator of all $V A$ events (B). AUC, area under the ROC curve; VA, ventricular tachyarrhythmia.
A

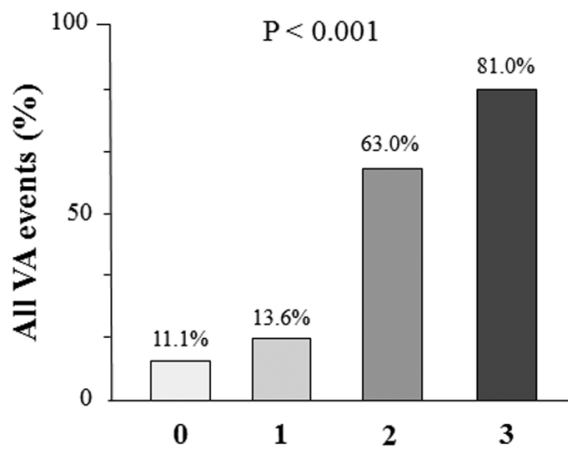

Localisation score

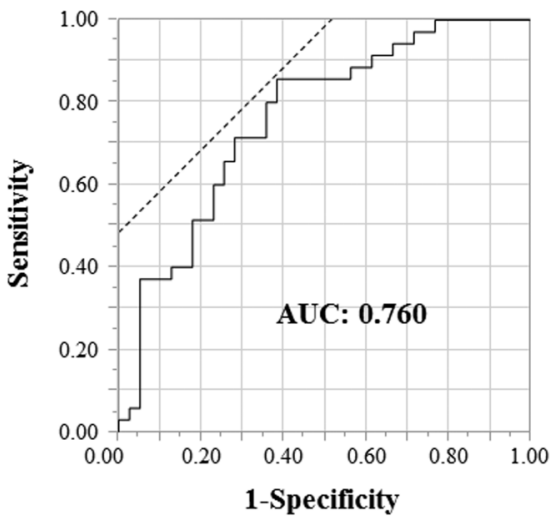

Fibrosis mass index
Figure 3 Kaplan-Meier analysis for the MACE-free (A and $B$ ) and VA-free survival $(C$ and $D)$ in patients stratified by baseline LV fibrosis mass index ( $A$ and $C$ ) and localisation score (B and $D)$. Lower group, solid line; higher group, dotted line. MACE, major adverse cardiac event; VA, ventricular tachyarrhythmia.
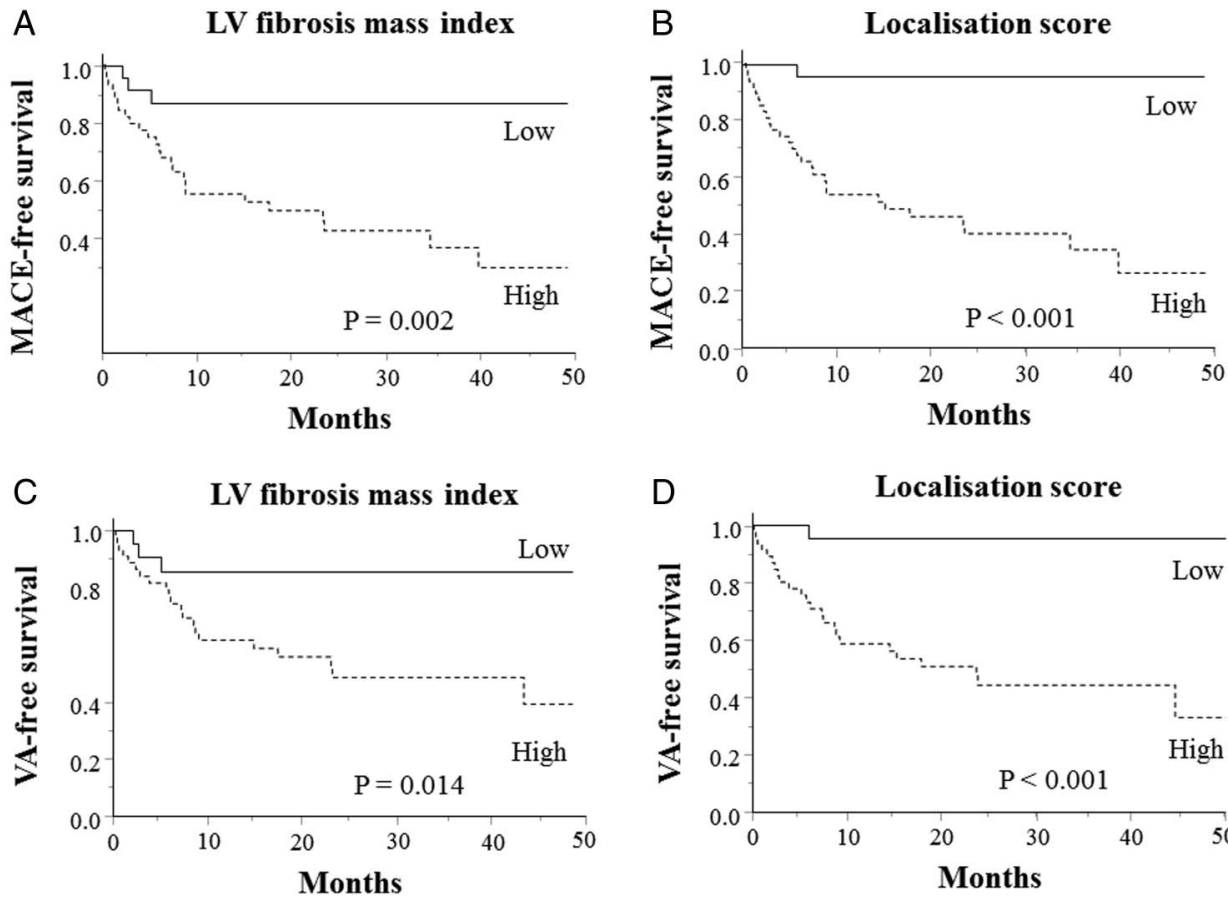

In multivariate Cox proportional hazard analysis with age, gender and FM index, the LS was independently associated with MACE and VA events, as shown in table 3. When the FM index as a continuous variable and the LS as throughout its range were entered into the analysis, the LS was still an independent predictor for MACE and VA $(\mathrm{P}<0.001$ and $\mathrm{P}=0.002$, respectively). Furthermore, as shown in figure 4 , the combined analysis for the higher FM index and LS groups showed that the combination was associated with the highest risk of MACE and VA events. Notably, all patients with admission for worsening HF or advanced AVB also belonged to this group. In contrast, patients with a FM index $<5.12 \mathrm{~g} / \mathrm{m}^{2}$ and an LS $\leq 1$ had no MACEs, or no VA events.

\section{Comparison between definite and suspected CS}

In comparison of baseline clinical characteristics between patients with definite and suspected CS, frequent extracardiac involvement and increased plasma ACE level were observed in definite CS $(p<0.01$, respectively) (see online supplementary table S1). Also, corticosteroid therapy was frequently performed $(\mathrm{p}=0.003)$. In CMR analysis, no significant differences were observed between them (see online supplementary table S2). In Kaplan-Meier analysis, each group also showed that the higher LS group $(\geq 2)$ had a worse prognosis in both MACE and VA events (figure 5).

\section{DISCUSSION}

The major findings of this study are as follows. First, VA was the most frequent cardiac event in CS, and VA events, including a prior history of VA, occurred in $48.1 \%$ of patients. Second, an LV FM index $\geq 5.12 \mathrm{~g} / \mathrm{m}^{2}$ was shown to predict MACE and VA. Third, a developed LS, defined as the summation of the LGE in the basal anterior LV, basal anteroseptal $\mathrm{LV}$ and $\mathrm{RV}$, was able to 
Table 3 Multivariate Cox proportional hazard analysis

\begin{tabular}{lccr}
\hline Variables & HR & $\mathbf{9 5 \%} \mathbf{C l}$ & $\mathbf{p ~ V a l u e ~}$ \\
\hline MACE & & & $<0.001$ \\
Age & 0.958 & 0.921 to 0.999 & 0.043 \\
Gender; male/female & 1.235 & 0.545 to 2.722 & 0.604 \\
LV fibrosis mass; high/low & 2.198 & 0.669 to 10.327 & 0.210 \\
$\quad$ Localisation score; high/low & 20.487 & 3.504 to 398.919 & $<0.001$ \\
VA events & & & $<0.001$ \\
Age & 0.987 & 0.946 to 1.035 & 0.580 \\
Gender; male/female & 1.525 & 0.620 to 3.685 & 0.350 \\
LV fibrosis mass; high/low & 1.308 & 0.395 to 6.098 & 0.685 \\
Localisation score; high/low & 14.546 & 2.579 to 276.708 & $<0.001$ \\
\hline MACE, major adverse cardiac events; VA, ventricular tachyarrhythmias. & &
\end{tabular}

predict VA when $\geq 2$. Fourth, the combination of both FM and LS improved the risk stratification for predicting clinical outcomes, including VA.

\section{Prognosis and VA in patients with CS}

In a retrospective autopsy series of SCD in sarcoidosis, 25 of 41 patients had evidence of cardiac involvement and 10 of 25 patients had no evidence of sarcoid in any other organ. ${ }^{15}$ Fleming et $a l^{16}$ reported that SCD occurred in 48 of 197 (24\%) patients with CS in their cohort. In a retrospective study of Japanese patients with CS, sustained VT was shown to be an independent risk factor for mortality, suggesting that VA is very important for prognosis of $\mathrm{CS}^{6}{ }^{6}$ In the present study, only two patients had SCD caused by a VA, or 12 patients had severe ventricular arrhythmias. We therefore tried to identify a better risk stratification tool for VA in CS that was more closely associated with SCD.

In an autopsy series of SCD in sarcoidosis, the ventricular septum had the largest percentage of involvement and $\sim 50 \%$ of the cases were involved in the RV apex and septum. ${ }^{15}$ Kumar et $a l^{17}$ reported that among 21 patients with CS and VT, 16 of 18 patients had an RV scar and 14 of 15 patients had an LV scar, with LV scarring frequently present in the septal $(11 / 15)$ and anterior $(7 / 15)$ LV. Similarly, our findings indicated LGE in basal septum at a high frequency among the patients with VAs (segment 2, 92.1\%; segment 3, 79.0\%), followed by the RV (73.7\%) and basal anterior $\mathrm{LV}$ (segment $1,60.5 \%$ ). In a recent cohort study of CS by Crawford et al, ${ }^{18}$ the presence of LGE in the RV was associated with adverse events, including VT/VF. Consistent with their studies, our results suggest that the presence of LGE in the RV may be important for the origin of VT.

For further risk stratification, we utilised a threesegment model (segment 1, 2 and RV), which showed highly significant differences in the presence of LGE between patients with and without VA (table 2). These were then used to define the LS, which was itself highly associated with not only all VA events (figure 2) but also follow-up VA events $(\mathrm{P}<0.001$, data not shown). The reason the LS was useful for risk stratification in VA is unclear. However, myocardial scar has been known to be an origin and a specific substrate of VA and it is proposed that the scars in the His-Purkinje system, LV outflow-tract in the basal septum and the anterior wall may participate in VA generation or SCD processes in CS using voltage cardiac mapping. ${ }^{17}$ Indeed, in the present study, the origin of sustained VT was estimated in 10 patients and the $80 \%$ was from left or right ventricular outflow tract or high LV septum, suggesting the close relationship between VT origin and LS (ie, the existence of LGE in segment 1, 2 or RV). With regard to the importance of $\mathrm{RV}$ involvement, Schuller et $a l^{19}$ reported that RV dysfunction, in particular, was associated with appropriate ICD therapy in patients with CS.
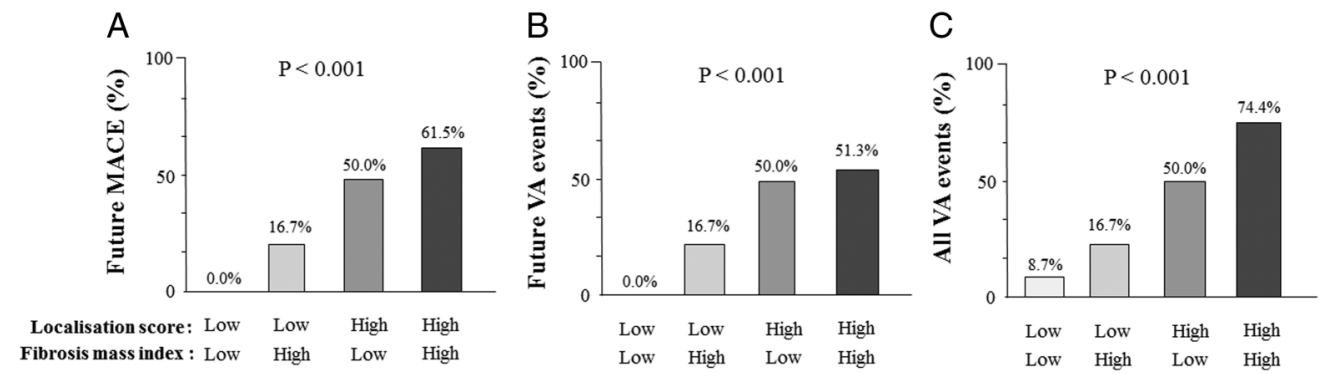

Figure 4 Risk stratification of clinical outcomes by the combined analysis of LV fibrosis mass and localisation score: MACE (A), VA events (B) and all VA events (a history of VA and new-onset VA events) (C). MACE, major adverse cardiac event; VA, ventricular tachyarrhythmia. 
Figure 5 Kaplan-Meier analysis for the MACE-free or VA-free survival in patients with definite CS ( $A$ and $C$ ) or suspected CS (B and D) stratified by localisation score. Lower group, solid line; higher group, dotted line. CS, cardiac sarcoidosis; MACE, major adverse cardiac event; VA, ventricular tachyarrhythmia.
A

Patients with definite CS ( $(n=35)$

Localisation score

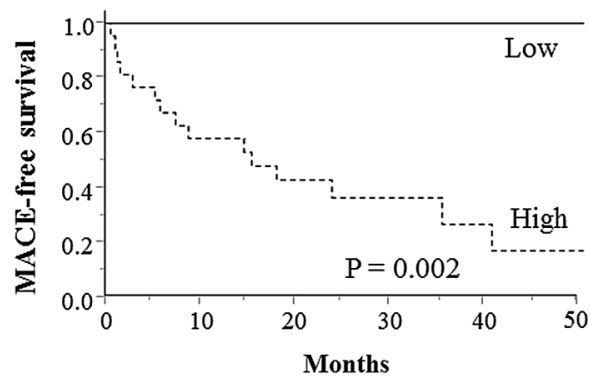

C

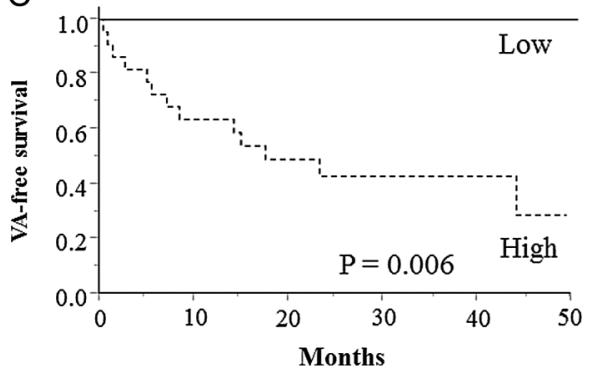

B

Patients with suspected CS ( $n=46)$ Localisation score

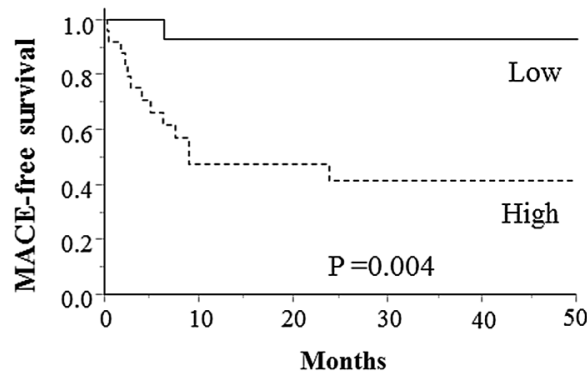

D

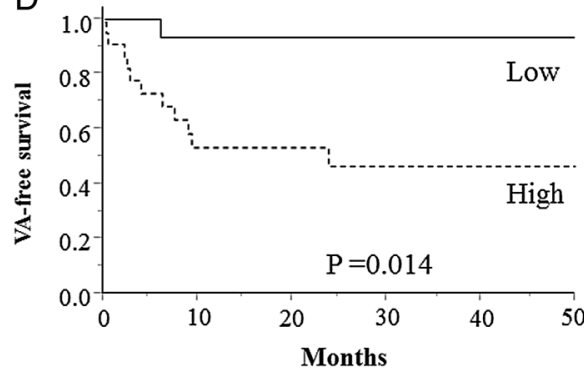

Further mechanistic studies for VA generation will clarify and improve the risk stratification in CS.

\section{LGE and VA in cardiomyopathies}

In studies of LGE in patients with VA, including both ischaemic and non-ischaemic cardiomyopathies, the presence of fibrosis was an independent predictor of adverse outcomes. $^{20}$ In a meta-analysis by Kuruvilla et $a l^{21}$, the presence and extent of LGE were associated with increased risk of all-cause mortality, HF hospitalisation, and SCD in patients with overall non-ischaemic cardiomyopathy. However, given that the basic pathophysiology is different for each cardiomyopathy, the relationship between LGE and each event may also diverge, suggesting that LGE quantification cannot be uniformly adapted to all cardiomyopathies. In a cohort study of 1293 patients with hypertrophic cardiomyopathy, the extent of LGE was associated with an increased risk of SCD, with the adjusted HR reported as 1.46 per $10 \%$ increase in LGE. ${ }^{22}$ In a study of dilated cardiomyopathy, mid-wall fibrosis was present in $142(30 \%)$ patients, and its assessment with LGE-CMR imaging provided independent prognostic information. ${ }^{23}$

Similarly, the presence of LGE among patients with suspected CS yielded a Cox HR of 31.6 for death, aborted SCD and appropriate ICD discharge, and of 33.9 for events including death and VT during a 3.4-year follow-up in a recent prospective study. ${ }^{9}$ However, they found no relationship between cardiac events including VA and LGE extent, the studied cohort consisted of a broad range of patients with systemic sarcoidosis, and the \% LGE in all patients was lower than that in our study cohort $(4.4 \%$ vs $16.6 \%$, respectively). In the present study, our cohort may be representative of more advanced CS, resulting in the increased LV FM by quantification being significantly associated with MACE or VA events by Kaplan-Meier analysis, and the ROC curve analysis showing an optimal cut-off value of $5.12 \mathrm{~g} /$ $\mathrm{m}^{2}$ in LV FM index for VA events. A recent study in 43 Japanese patients with CS taking steroid therapy, where $\%$ LGE was $19 \% \pm 10 \%$ (5-SD method), showed that large-extent LGE (ie, \% LGE $\geq 20 \%$ ) was independently associated with combined adverse outcomes. ${ }^{24}$ Crawford et $a l^{18}$ also reported that LGE involving $\geq 9$ segments in a combined left and right ventricular segmental analysis resulted in a $92 \%$ sensitivity and $88 \%$ specificity for differentiating patients with VT/VF from those without VT/VF (AUC, 0.90). In addition, the results of the present study suggest an incremental effect of the LS on LGE extent (LV FM) by LGE assessment, and that a combined analysis of LS and LV FM may be better for the risk stratification of clinical outcomes in CS.

\section{Study limitations}

Several limitations should be considered when interpreting our results. First, the study population was relatively small and could have resulted in low statistical power. However, the size of our cohort was average relative to that of other studies of CMR in CS. ${ }^{17} 18$ Also, the retrospective nature of the study may suggest some impact of the missing or insufficient data on the present results.

Second, about half of the patients did not fulfil the JMHW diagnostic criteria (suspected CS), most of whom had isolated CS. Recently, several reports have suggested that there are similar clinical characteristics and clinical outcomes for suspected CS that does not fulfil the JMHW diagnostic criteria but that satisfies only the guidelines for clinical cardiac diagnosis. ${ }^{25}$ To test this, we analysed and compared the data between the patients who did (definite CS) and did not fulfil the 
JMHW diagnostic criteria (suspected CS), and found that the clinical characteristics were indeed similar and that the higher LS group $(\geq 2)$ had a worse prognosis in each group (figure 5).

Third, the study population consisted of patients with CS who could undergo CMR. In particular, most patients with previously implanted cardiac devices and those with severe renal failure were not included in the present study since they could not tolerate the procedure. Therefore, the event rate and clinical characteristics might be underestimated in the present analysis.

Contributors MY and YI are the primary investigators. TK, TI, YI, TN, TK, YM are the secondary investigator (data sampling/analysis). SI, M I, TK, SM are consultants and supervisors.

Competing interests None.

Ethics approval Kindai University Faculty of Medicine.

Provenance and peer review Not commissioned; externally peer reviewed.

Open Access This is an Open Access article distributed in accordance with the Creative Commons Attribution Non Commercial (CC BY-NC 4.0) license, which permits others to distribute, remix, adapt, build upon this work noncommercially, and license their derivative works on different terms, provided the original work is properly cited and the use is non-commercial. See: http:// creativecommons.org/licenses/by-nc/4.0/

\section{REFERENCES}

1. Silverman KJ, Hutchins GM, Bulkley BH. Cardiac sarcoid: a clinicopathologic study of 84 unselected patients with systemic sarcoidosis. Circulation 1978;58:1204-11.

2. Longcope WT, Freiman DG. A study of sarcoidosis; based on a combined investigation of 160 cases including 30 autopsies from The Johns Hopkins Hospital and Massachusetts General Hospital. Medicine (Baltimore) 1952;31:1-132.

3. Kim JS, Judson MA, Donnino R, et al. Cardiac sarcoidosis. Am Heart J 2009;157:9-21.

4. Doughan AR, Williams BR. Cardiac sarcoidosis. Heart 2006;92:282-8.

5. Roberts WC, McAllister HA Jr, Ferrans VJ. Sarcoidosis of the heart. A clinicopathologic study of 35 necropsy patients (group 1) and review of 78 previously described necropsy patients (group 11). Am J Med 1977;63:86-108.

6. Yazaki $\mathrm{Y}$, Isobe M, Hiroe M, et al. Prognostic determinants of long-term survival in Japanese patients with cardiac sarcoidosis treated with prednisone. Am J Cardiol 2001;88:1006-10.

7. Smedema JP, Snoep G, van Kroonenburgh MP, et al. Evaluation of the accuracy of gadolinium-enhanced cardiovascular magnetic resonance in the diagnosis of cardiac sarcoidosis. J Am Coll Cardiol 2005;45:1683-90.

8. Ohira $\mathrm{H}$, Tsujino I, Ishimaru $\mathrm{S}$, et al. Myocardial imaging with 18-F-fluoro-2-deoxyglucose positron emission tomography and magnetic resonance imaging in sarcoidosis. Eur $\mathrm{J} \mathrm{Nucl} \mathrm{Med} \mathrm{Mol}$ Imaging 2008;35:933-41.

9. Greulich S, Deluigi CC, Gloekler S, et al. CMR imaging predicts death and other adverse events in suspected cardiac sarcoidosis. JACC Cardiovasc Imaging 2013;6:501-11.

10. Tahara N, Tahara A, Nitta Y, et al. Heterogeneous myocardial FDG uptake and the disease activity in cardiac sarcoidosis. JACC Cardiovasc Imaging 2010;3:1219-28.

11. Youssef $\mathrm{G}$, Beanlands RS, Birnie $\mathrm{DH}$, et al. Cardiac sarcoidosis: applications of imaging in diagnosis and directing treatment. Heart 2011;97:2078-87.

12. Tadamura E, Yamamuro M, Kubo S, et al. Effectiveness of delayed enhanced MRI for identification of cardiac sarcoidosis: comparison with radionuclide imaging. AJR Am J Roentgenol 2005;185:110-5.

13. Nakamura T, Iwanaga $Y$, Yasuda M, et al. Relationship of basal-septal fibrosis with LV outflow tract obstruction in hypertrophic cardiomyopathy: insights from cardiac magnetic resonance analysis Int J Cardiovasc Imaging 2016;32:613-20.

14. Flett AS, Hasleton J, Cook C, et al. Evaluation of techniques for the quantification of myocardial scar of differing etiology using cardiac magnetic resonance. JACC Cardiovasc Imaging 2011;4:150-6.

15. Tavora F, Cresswell N, Li L, et al. Comparison of necropsy findings in patients with sarcoidosis dying suddenly from cardiac sarcoidosis versus dying suddenly from other causes. Am J Cardiol 2009;104:571-7.

16. Fleming HA, Bailey SM. Sarcoid heart disease. J R Coll Physicians Lond 1981;15:245-6, 249-53.

17. Kumar S, Barbhaiya C, Nagashima K, et al. Ventricular tachycardia in cardiac sarcoidosis: characterization of ventricular substrate and outcomes of catheter ablation. Circ Arrhythm Electrophysiol 2015;8:87-93.

18. Crawford T, Mueller G, Sarsam S, et al. Magnetic resonance imaging for identifying patients with cardiac sarcoidosis and preserved or mildly reduced left ventricular function at risk of ventricular arrhythmias. Circ Arrhythm Electrophysiol 2014;7:1109-15.

19. Schuller JL, Zipse M, Crawford T, et al. Implantable cardioverter defibrillator therapy in patients with cardiac sarcoidosis. J Cardiovasc Electrophysiol 2012;23:925-9.

20. Dawson DK, Hawlisch K, Prescott G, et al. Prognostic role of CMR in patients presenting with ventricular arrhythmias. JACC Cardiovasc Imaging 2013:6:335-4.

21. Kuruvilla S, Adenaw N, Katwal AB, et al. Late gadolinium enhancement on cardiac magnetic resonance predicts adverse cardiovascular outcomes in nonischemic cardiomyopathy: a systematic review and meta-analysis. Circ Cardiovasc Imaging 2014;7:250-8.

22. Chan $\mathrm{RH}$, Maron BJ, Olivotto I, et al. Prognostic value of quantitative contrast-enhanced cardiovascular magnetic resonance for the evaluation of sudden death risk in patients with hypertrophic cardiomyopathy. Circulation 2014;130:484-95.

23. Gulati A, Jabbour A, Ismail TF, et al. Association of fibrosis with mortality and sudden cardiac death in patients with nonischemic dilated cardiomyopathy. JAMA 2013;309:896-908.

24. Ise $\mathrm{T}$, Hasegawa $\mathrm{T}$, Morita $\mathrm{Y}$, et al. Extensive late gadolinium enhancement on cardiovascular magnetic resonance predicts adverse outcomes and lack of improvement in LV function after steroid therapy in cardiac sarcoidosis. Heart 2014;100:1165-72.

25. Takaya Y, Kusano KF, Nakamura K, et al. Comparison of outcomes in patients with probable versus definite cardiac sarcoidosis. $A m$ J Cardiol 2015;115:1293-7. 